\title{
Dried Plasmodium falciparum-infected samples as positive controls for malaria rapid diagnostic tests
}

\author{
Michael Aidoo*, Jaymin C Patel and John W Barnwell
}

\begin{abstract}
Background: Rapid diagnostic tests (RDTs) are central to fulfilling the WHO's recommendation for parasitologic confirmation of all suspected cases of malaria. RDT performance may be compromised when exposed to the high temperature conditions typical of most malaria endemic regions. However, a systematic method to monitor RDT quality and performance in endemic countries is lacking at the present time. Current methods to monitor RDT performance in the field include comparing results from RDTs to diagnoses made by light microscopy and observing health workers perform tests. These methods are not substitutes for direct quality control. In this study, the suitability of dried Plasmodium falciparum-infected blood as quality control samples for malaria RDTs was evaluated.
\end{abstract}

Methods: Three cultured strains of $P$. falciparum at 200 and 2,000 parasites/ $\mu$ l were tested on 10 brands of RDT. After baseline testing to determine initial reactivity, aliquots of parasite-infected blood were air dried, stored at $35^{\circ} \mathrm{C}$, room temperature $\left(\sim 25^{\circ} \mathrm{C}\right)$ or $4^{\circ} \mathrm{C}$ for one, four and 12 weeks and were then tested on the 10 RDTs after rehydration. Extended stability testing of dried blood stored at $4^{\circ} \mathrm{C}$ was done using $P$. falciparum strain $3 D 7$ at 1,000 and 2,000 parasites/ $\mu$ l.

Results: All dried blood samples at 2,000 parasites/ $\mu$ l retained reactivity (100\% sensitivity) at all three temperatures and time points for all nine RDT brands that detect histidine-rich protein-2 (HRP2). The dried blood samples with 200 parasites/ $\mu$ l were detected by six of the nine HRP2-based RDTs at all storage temperatures and time points. The sensitivity for two of the three remaining HRP2-based RDTs was 100\% up to four weeks of storage at all temperatures but dropped to $87.5 \%$ at week 12 . Of the four RDTs that detect plasmodium lactate dehydrogenase $(\mathrm{pLDH})$ in a pan-specific manner, alone or in combination with HRP2, the detection of pLDH in samples with 2,000 parasites/ $\mathrm{LL}$ was $100 \%$ for two RDTs and $80 \%$ for the other two RDTs. The mean level for detection of pLDH at 200 parasites/ $\mu$ l was low (29\%), with a range of $0 \%$ to 100\%, which was partly attributable to weak initial baseline reactivity. Reactivity of dried $3 \mathrm{D} 7$ at 1,000 and 2,000 parasites $/ \mu$ stored at $4^{\circ} \mathrm{C}$ was retained at $100 \%$ for up to 52 weeks for both HRP2 and pLDH.

Conclusions: In the absence of native or recombinant positive control antigens, well-standardized $P$. falciparuminfected dried blood samples can be used as positive control samples for monitoring RDT performance, particularly with HRP2-detecting tests.

\section{Background}

Malaria remains a serious health problem for much of the global population. Over 200 million cases of Plasmodium falciparum occur annually worldwide for which prompt treatment is required to prevent death, especially in children under the age of five. The burden of malaria

\footnotetext{
*Correspondence: maidoo@cdc.gov

Malaria Branch, Division of Parasitic Diseases and Malaria, Center for Global Health, US Centers for Disease Control and Prevention, 1600 Clifton Road, Atlanta, GA 30333, USA
}

(c) 2012 Aidoo et al.; licensee BioMed Central Ltd. This is an Open Access article distributed under the terms of the Creative Commons Attribution License (http://creativecommons.org/licenses/by/2.0), which permits unrestricted use, distribution, and reproduction in any medium, provided the original work is properly cited. is highest in sub-Saharan Africa. However, travellers from malaria non-endemic countries are also at increased risk of severe disease if exposed to this parasite. Accurate diagnosis of malaria is critical to administering appropriate treatment. In the past, due to the high prevalence of malaria among febrile patients and the availability of cheap anti-malarial drugs, malaria was diagnosed on the basis of clinical symptoms, with only a small proportion of cases confirmed with laboratory tests. However, the World Health Organization (WHO) 
updated malaria treatment guidelines in 2010 to emphasize parasitologic confirmation of all suspected cases [1]. This decision was made for multiple reasons, including recent reductions in the incidence of malaria in many endemic countries [2-5], the spread of parasite resistance requiring a switch to more expensive artemisininbased combination therapy (ACT) and the need to reduce drug pressure to prevent development and spread of resistance to ACT.

Traditionally, malaria diagnosis has relied on light microscopic examination of stained blood smears. However, the capacity to conduct quality routine malaria microscopy has been low, resulting in little or no use of the laboratory to confirm suspected cases and a mistrust of laboratory test results by clinicians [6,7]. In recent years, malaria rapid diagnostic tests (RDTs) have been developed and shown to be comparable or surpass routine microscopy when the use of quality controlled, well performing test kits [8] is coupled with adequate training and carefully designed bench aids [9-12]. RDTs are simple and can be used by non-laboratory staff in health facilities and by community health workers [10-12]. The performance of quality controlled RDTs and their ease of use by a wide variety of non-laboratory health workers have also, in part, led to the new WHO recommendation on parasitologic confirmation of all suspected cases.

Despite their utility, RDTs have several limitations that need to be considered prior to their routine use. First, RDT products from different manufacturers can differ widely in performance characteristics especially in their ability to identify low parasite density infections, and inter-lot variation among some tests from the same manufacturer have been reported [13]. In addition, RDT performance can be compromised when tests are stored for long periods at high temperatures and humidity typical of most malaria-endemic countries [14]. Ensuring good manufacturing quality is addressed to an extent by the RDT product evaluation [13] and pre-procurement lot testing programmes conducted by the WHO, Foundation for Innovative New Diagnostics (FIND) and US Centers for Disease Control and Prevention (CDC). Data from these programmes are available to national malaria control programmes and are intended to ensure the purchase of quality RDTs from manufacturers. In addition, the WHO provides criteria for selecting RDTs with different sensitivities at low parasite densities that is based on malaria endemicity. However, quality control (QC) of RDTs in the field after delivery remains a major challenge. No systematic method exists to monitor the performance of RDTs at the point of care. Such RDT performance monitoring in the field is essential since tests are affected by storage under conditions not specified by manufacturers. One method of QC employed in the field has been to compare results from RDTs to results from light microscopic examination of stained blood smears from the same clinical specimen. This is not an ideal comparison as the two tests detect parasites differently; RDTs detect parasite antigens by immunochromatographic methods whereas microscopy detects whole parasites. As a result of this difference and because parasite antigens, especially HRP2, persist for several days after parasite clearance, RDT positive tests could be negative by smear microscopy in cases where patients have received anti-malarial treatment within a few days or even weeks of being tested $[15,16]$. Furthermore, accurate slide reading requires proficient microscopists unavailable in most peripheral health facilities in sub-Saharan Africa [6,17]. The second method of observing how well health workers perform RDTs assures the competence of the health worker only and not the quality of the test since the reactivity of patient samples are unknown. Recombinant antigens are being developed for malaria RDTs [18,19]; however, it is not clear when such antigens will be available for use in the field. A method using dried blood spots on filter paper showed some promise; however, the method was not simple, requiring elution of blood from filter paper without a clear correlation of parasite density in the eluted sample to the density in the original sample [20]. In addition, only one culture-derived parasite was tested on three RDT brands, which according to the WHO/FIND Round 2 RDT product testing report had low performance characteristics. Frozen parasite isolates have been used however; the need for freezing parasites at temperatures below $-70^{\circ} \mathrm{C}$ makes the method impractical for most resource-limited malaria endemic settings.

Millions of malaria RDTs are currently being used in malaria endemic countries without suitable methods for QC at the point of care. While the field waits for recombinant antigens, a simple method of using dried parasitized blood in tubes adapted from a similar method used for HIV rapid tests [21] was developed for malaria rapid tests. Here, an evaluation of the method and how it can be used for malaria RDT QC is reported.

\section{Methods}

\section{Rapid diagnostic test choice}

RDTs were chosen based on performance in Round 2 of WHO/FIND/CDC RDT performance evaluations [13] and their availability for purchase (Table 1). Performance criteria used for test selection was a panel detection score (PDS) at 200 parasite/ $\mu \mathrm{l}$ of $\geq 90$ for Plasmodium falciparum. PDS for Plasmodium vivax was not considered for selection since only P. falciparum samples were available for this study. Based on the above criterion, 10 RDTs were selected for this study (Table 1). Four of the tests (RDTs 1, 4, 7 and 9) were specific for $P$. falciparum 
only; with three detecting HRP2 only and the fourth detecting both HRP2 and P. falciparum-specific pLDH. Three tests (RDTs 2, 6 and 10) were combination tests detecting HRP2 and pan- specific pLDH. Two other combination tests (RDTs 3 and 8) detected P. falciparum and $P$. vivax by HRP2 and $P$. vivax-specific $\mathrm{pLDH}$ respectively. One test (RDT 5) was a combination test that recognized Plasmodium species by detecting panspecific $\mathrm{pLDH}$. The same lot of each RDT kit was used for all experiments with the exception of RDT 1. For this RDT the same lot was used for baseline, week 1 and 4 experiments and a different lot used for week 12 experiments.

\section{Dried Plasmodium falciparum-infected blood preparation}

Three in vitro-cultured P. falciparum strains, Nigeria XII, PH 1, and FC27/A3 were used for this study. These parasites were stored as frozen $50 \mu$ l aliquots standardized at 200 or 2,000 parasites/ $\mu$ l. Parasites were at ring stage development $(>95 \%)$ and parasite levels were determined and standardized by established methods [22]. A $50 \mu \mathrm{l}$ aliquot of frozen blood at 200 and 2,000 parasites/ $\mu$ l for each of the three $P$. falciparum strains was thawed and tested for baseline reactivity. The frozen vials were thawed at room temperature, mixed briefly and tested on each of the 10 RDT brands. Replicate aliquots were then air dried overnight in a bio-safety cabinet. After checking that samples were completely dry, vials were sealed by tightly closing the vial cap and stored in a refrigerator set at $4^{\circ} \mathrm{C}$, at room temperature $\left(\sim 25^{\circ} \mathrm{C}\right)$ or in a dry incubator set at $35^{\circ} \mathrm{C}$. A schematic diagram of dried blood preparation is shown in Figure 1.

\section{Dried blood rehydration and rapid diagnostic testing}

Dried blood pellets in tubes were rehydrated with PBSTween [one sachet of PBS-Tween 20 (SIGMA, USA) dissolved in 1 litre of de-ionized water yielding $0.01 \mathrm{M}$
PBS; $0.138 \mathrm{M} \mathrm{NaCl} ; 0.0027 \mathrm{M} \mathrm{KCl}$ and $0.05 \%$ Tween 20 at a $\mathrm{pH}$ of 7.4]. On the day of testing, one vial of each of the three parasite isolates at 200 and 2,000 parasites/ $\mu \mathrm{l}$ and at each storage temperature was rehydrated with $50 \mu \mathrm{l}$ (same as initial blood volume before drying) of PBS-Tween and left at room temperature for a minimum of $1 \mathrm{hr}$. Samples were then gently mixed with the use of a micropipette. RDT testing was performed according to the manufacturer's instructions for each brand. The only exception was the use of a micropipette rather than the test-supplied blood transfer device to transfer $5 \mu \mathrm{l}$ of blood from the tube onto the test. Samples were tested after one, four and 12 weeks of storage. At each time point, each of the three isolates at 200 and 2,000 parasites $/ \mu \mathrm{l}$ was tested for the three storage temperatures (total of 18 tests) except for room temperature-stored Nigeria XII for weeks 4 and 12 and PH1 at 2,000 parasites/ $\mu$ l for all temperatures at week 12 and $35^{\circ} \mathrm{C}$ at week 4 . The former was due to a laboratory error that led to the samples being reconstituted at week 1 and the latter due to unavailability of sufficient number of aliquots. For the purposes of this investigation, a weak reactivity is when a band is distinctly faint when compared to the control band and required brighter light and a second reader to confirm the presence of a band. No densitometry measurements were used to determine band intensity. Band intensity for initial testing of the 10 RDT brands was scored subjectively. However, a band intensity scale became available for scoring of test bands in the extended stability testing and, therefore, an objective "+" scoring system was used. A false negative test was defined as P. falciparum-infected blood identified as positive by a test at baseline (before drying) and appearing as a negative test after drying irrespective of storage temperature. For combination tests, the HRP2 and pLDH bands were considered separately.

Table 1 Rapid diagnostic test type and antigen specificity for the 10 RDTs used in testing dried blood samples

\begin{tabular}{|c|c|c|c|c|c|}
\hline Product & $\begin{array}{l}\text { Number } \\
\text { of bands* }\end{array}$ & $\begin{array}{l}\text { Parasite } \\
\text { specificity }\end{array}$ & Manufacturer & $\begin{array}{l}\text { Product } \\
\text { code }\end{array}$ & $\begin{array}{l}\text { Pf PDS\# @ } \\
200 \text { parasites/ } \mu \mathrm{l}\end{array}$ \\
\hline CareStart $^{\mathrm{TM}}$ Malaria HRP2/pLDH Pf test & 2 & Pf & Access Bio, Inc. & G0181 & 98 \\
\hline CareStart Malaria HRP2/pLDH (Pf/PAN) COMBO & 3 & Pf/Pan & Access Bio, Inc. & G0131 & 97 \\
\hline 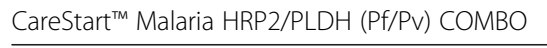 & 3 & $\mathrm{Pf} / \mathrm{Pv}$ & Access Bio, Inc. & G0161 & 90 \\
\hline CareStart Malaria HRP2 (Pf) & 2 & Pf & Access Bio, Inc. & G0141 & 99 \\
\hline CareStart Malaria pLDH (PAN) & 2 & Pan & Access Bio, Inc. & G0111 & 92 \\
\hline First Response Malaria Ag Combo (PLDH/HRP2) & 3 & Pan & Premier Medical Corporation Ltd & II6FRC30 & 100 \\
\hline First Response Malaria Ag HRP2 & 2 & Pf & Premier Medical Corporation Ltd & II3FRC30 & 100 \\
\hline SD BIOLINE Malaria Ag Pf/Pv (HRP2/pLDH) & 3 & $\mathrm{Pf} / \mathrm{Pv}$ & Standard Diagnostics, Inc. & 05FK80 & 96 \\
\hline SD BIOLINE Malaria Ag Pf (HRP2) & 2 & Pf & Standard Diagnostics, Inc. & 05FK50-02-4 & 97 \\
\hline SD BIOLINE Malaria Ag Pf/Pan (HRP2/pLDH) & 3 & Pf/Pan & Standard Diagnostics, Inc. & 05FK60-02- 3 & 96 \\
\hline
\end{tabular}

* Number of bands including controls.

\# Panel Detection Score from WHO Malaria RDT Product Testing Round 2. 


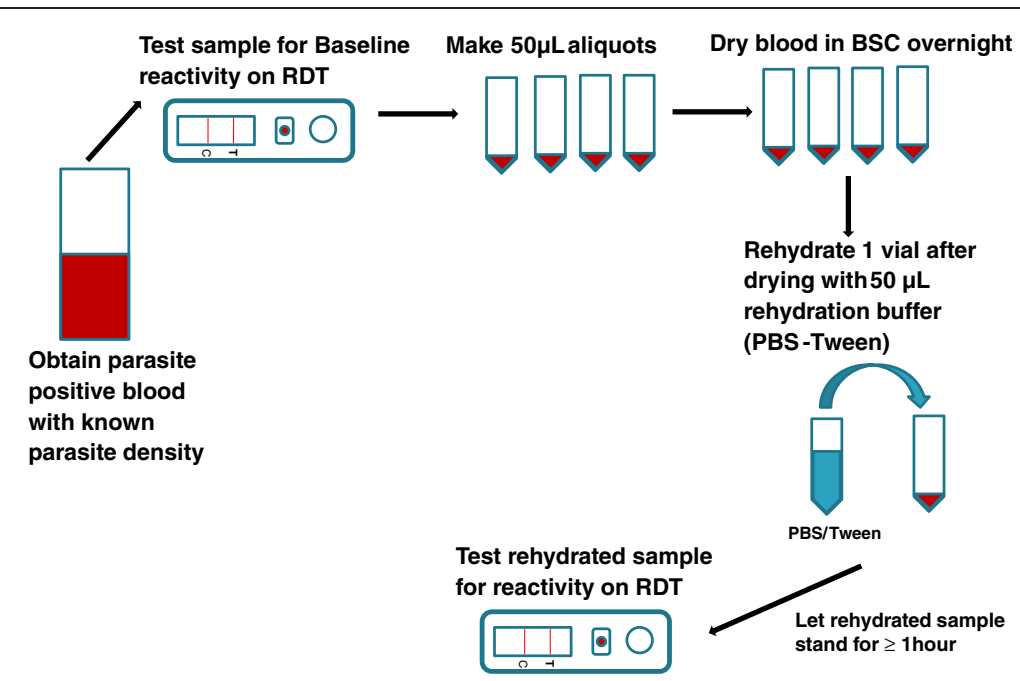

Figure 1 Schematic diagram for preparing dried blood tubes for malaria RDT QC.

\section{Sensitivity calculation}

Sensitivity was calculated using the baseline test result on a particular test before drying as the reference gold standard. Therefore sensitivity for dried blood detection was calculated to be product specific. Furthermore, because storage temperature appeared not to influence test results for the 12-week period, sensitivity of dried blood detection was calculated by combining tests results from tubes stored at the three storage temperatures. For combination tests, sensitivity was calculated separately for the HRP2 (Pf) and pLDH (Pan) bands, independently for each product that detected these antigens.

\section{Dried blood stability}

To determine the stability of dried blood over time, a panel of samples using P. falciparum clone 3D7 at 1,000 and 2,000 parasites/ $\mu$ l was produced. Cultured 3D7 containing rings or early trophozoite forms were produced using standard malaria culture procedures. A Giemsastained smear read by three microscopists was used to determine parasitaemia calculated by the number of infected cells per 1,000 red blood cells. A Coulter Counter $^{\circledR}$ (Beckman-Coulter, Brea, California, USA) was used to determine the number of erythrocytes/ $\mu$ l and the number of parasites/ $\mu \mathrm{l}$ subsequently determined from the total number of $\mathrm{RBCs} / \mu \mathrm{l}$. Uninfected blood with a similar haematocrit was used to dilute the cultured parasites to 1,000 and 2,000 parasites/ $\mu$ l. The parasite dilutions obtained were then tested on RDTs 2, 5 and 10 to determine baseline reactivity and then distributed into $60 \mu \mathrm{l}$ aliquots in tubes, dried as described above and stored at $4^{\circ} \mathrm{C}$. At weeks 12, 21, 26, 35, 38, 41, and 52 following dried-tube preparation, vials at 1,000 and 2,000 parasites/ $\mu \mathrm{l}$ were rehydrated with $60 \mu \mathrm{l}$ PBS-Tween and tested on RDT 2 for up to 52 weeks and
RDT 5 for up to 46 weeks. Testing was also done on RDT 10 for up to 26 weeks for samples with 1,000 parasites/ $\mu \mathrm{l}$ and up to 21 weeks for samples with 2,000 parasites/ $\mu$ l. RDTs 1,3 and 7 were also tested only at week 52 .

Time series experiments using punctured RDT pouches

To simulate the ability of dried blood to identify faulty RDTs, an experiment was designed in which two sets each of RDTs 2 and 5 were stored either at room temperature $\left(\sim 25^{\circ} \mathrm{C}\right)$ or in a humidified incubator set at $37^{\circ} \mathrm{C}$. One set each of RDTs at each storage temperature had two $8 \mathrm{~mm}$ holes punctured on each side of the test pouch with the desiccant intact. At 2 hrs, $6 \mathrm{hrs}, 30 \mathrm{hrs}, 2$ weeks and 4 weeks, duplicate RDTs were taken from each set and tested using reconstituted dried blood (3D7) at 1000 parasites/ $\mu$ l. Punctured tests stored at room temperature for both RDT2 and RDT5 were not tested at weeks 2 and 4 due to unavailability of tests.

\section{Results}

Rapid diagnostic test brand-specific reactivity

Overall, four of the 10 RDT brands (RDTs 1, 3, 4, and 8), all HRP2-specific tests, maintained reactivity that was comparable to baseline with dried blood specimens at both parasite concentrations of 200 and 2,000 parasites/ $\mu \mathrm{l}$ held at all storage temperatures and time points tested (Additional file 1: Supplementary information a, c, d, h). Two RDT brands (RDT 7 and 9), both HRP2 tests, also maintained reactivity for all samples at both parasite concentrations at all temperatures and time points with the exception of specimens of the PH 1 strain at 200 parasites/ $\mu$ l when stored at $35^{\circ} \mathrm{C}$ for 12 weeks (Additional file 1: Supplementary information $\mathrm{g}$ and i). The remaining four RDT brands (RDT 2, 5, 6 and 10; Additional file 1: 
Supplementary information b, e, f, j), which lost reactivity in 53 of 506 tests (10.5\% false negative), shared certain common characteristics. First, all four tests were combination tests designed to detect $P$. falciparum and the other three human malaria species. Second, with the exception of Nigeria XII parasites at 2,000 parasites/ $\mu \mathrm{l}$ (week 1, RT; week $4,35^{\circ} \mathrm{C}$ and week $12,35^{\circ} \mathrm{C}$ ) that were non-reactive with pLDH on RDT 6 (Additional file 1: Supplementary information $\mathrm{f}$ ), reactivity was lost only for samples at 200 parasites/ $\mu$ l. In addition, for three of four RDTs that detected $P$. falciparum separately from other plasmodium parasites (HRP2 and pLDH as separate bands), reactivity was lost for pLDH and not HRP2 for 2 RDTs (RDTs 2 and 10$)$ at the low (200 parasites/ $\mu$ l) parasite density. However, three samples of PH 1 at 200 parasites/ $\mu$ l were non-reactive for HRP2 on RDT 6, (Additional file 1: Supplementary information f). Finally, weak baseline reactivity of the pLDH band was the single most predictive factor for loss of reactivity after drying blood. The latter characteristic is particularly exemplified by RDT 5 , a pLDH only test, in which weak baseline band intensity for all three parasite strains at 200 parasites $/ \mu \mathrm{l}$ was predictive of non-reactivity after drying (Additional file 1: Supplementary information e).

\section{Effect of storage temperature and duration of storage on dried blood reactivity}

Storage temperature did not appear to significantly influence the reactivity of the dried blood. For RDTs 1, 3, 4 and 8 , no loss in reactivity compared to baseline was observed. In some instances, indications of storage temperature influencing dried blood reactivity could not be confirmed. Loss of reactivity for one parasite isolate stored at $35^{\circ} \mathrm{C}$ was not associated with a loss of reactivity for another parasite stored at the same temperature and length of storage. For example, RDT 5 at week 1, using dried PH 1 at 200 parasites/ $\mu \mathrm{l}$ was non-reactive for samples stored at $4^{\circ} \mathrm{C}$ and $\mathrm{RT}$ but reactive for the sample stored at $35^{\circ} \mathrm{C}$. At week 12 , the same sample was nonreactive for samples stored at $4^{\circ} \mathrm{C}$ and $35^{\circ} \mathrm{C}$ but reactive for the sample stored at RT. Another example is shown by parasite strain $\mathrm{FC} 27 / \mathrm{A} 3$ at 200 parasites/ $\mu \mathrm{l}$ on RDT5. At week 4, dried blood specimens stored at $4^{\circ} \mathrm{C}$ and $35^{\circ} \mathrm{C}$ were non-reactive while that stored at $\mathrm{RT}$ was reactive. Considering the weak baseline reactivity of both PH 1 and FC27/A3 at 200 parasites/ $\mu$ l on RDT 5, a pLDH-specific test, it is likely the loss of reactivity was due to the sample being detected close to the limit of detection of the test and not due to storage temperature. A stronger indication of an influence of storage temperature on dried blood reactivity is given by the reactivity of $35^{\circ} \mathrm{C}$-stored $\mathrm{PH} 1$ at 200 parasites/ $\mu \mathrm{l}$ at week 12 on RDTs 7 and 9, both HRP2-specific tests. This was the only non- reactive sample of all parasite/storage temperature/time combinations for these two RDTs.

\section{Effect of parasite isolate on dried blood reactivity}

At comparable parasite densities, similarly stored Nigeria XII and FC27/A3 but not PH 1 retained their reactivity on eight of 10 RDTs (RDTs 1, 3, 4, 5, 7, 8, 9 and 10); implying a parasite-specific reactivity profile. Indeed, at 200 parasites/ $\mu \mathrm{l}$, baseline reactivity for $\mathrm{PH} 1$ was weak or negative on RDTs 2, 5, 6 and 10. In addition, at 200 parasites/ $\mu \mathrm{l}$, PH 1 reactivity was weak for all storage temperatures at weeks 1 and 4 and for $4^{\circ} \mathrm{C}$ and $\mathrm{RT}$ at week 12 on RDT9 (Additional file 1: Supplementary information i). On RDT 7, weak reactivity was observed for this sample stored at $4^{\circ} \mathrm{C}$ for week 1 and for RT and $35^{\circ} \mathrm{C}$ for both weeks 4 and 12 . At week 12 , reactivity was lost for the sample stored at $35^{\circ} \mathrm{C}$. In addition to the reactivity on RDTs 7 and 9, weak reactivity for PH 1 at 200 parasites $/ \mu \mathrm{l}$ was observed for several samples on RDTs 2, 3, 4, 5, 6, 8 and 10.

\section{Sensitivity of dried blood detection}

Using the baseline result as the gold standard and combining results from all three parasites and storage conditions, all dried blood at 2,000 parasites/ $\mu$ l retained reactivity ( $100 \%$ sensitivity) on all nine RDT brands that detect HRP2 (Table 2). At 2,000 parasites/ $\mu$ l, sensitivity for pLDH (Pan) for two of four combination tests was $100 \%$. The sensitivities for the two remaining RDTs (RDTs 2 and 6) were lower at $80 \%$.

At 200 parasites/ $\mu \mathrm{l}$, dried blood stored at all temperatures and time points were detected at $100 \%$ sensitivity on six of nine RDTs for HRP2. The sensitivity on two of the three remaining HRP2 detecting RDTs was 100\% up to four weeks of storage at all temperatures, dropping to $87.5 \%$ for week 12 samples stored at $35^{\circ} \mathrm{C}$. The mean sensitivity of dried blood detection for pLDH was low (29\%, range $0-100 \%)$ at 200 parasites/ $\mu$ l. This low sensitivity was partly attributable to weak baseline reactivity on the pLDH bands on specific RDT brands. The experiments in this study were not designed to detect false positivity. Nevertheless, for the two RDTs specific for both $P$. falciparum and $P$. vivax, irrespective of storage condition, parasite density and length of storage, in no instance was a $P$. vivax band reactive.

\section{Dried blood stability}

Dried 3D7 at 1,000 and 2,000 parasites/ $\mu$ stored at $4^{\circ} \mathrm{C}$ remained reactive after several weeks of storage (Table 3a). RDT 2 (HRP2/pLDH) was reactive for both parasite densities for up to 52 weeks irrespective of the target antigen, although as expected, test band intensities for HRP2 were higher than for pLDH. Because of the unavailability of additional tests, RDT 5 (pLDH) 
Table 2 Sensitivity of detection for dried blood specimens at different parasite densities and storage times

\begin{tabular}{|c|c|c|c|c|}
\hline & & \multicolumn{3}{|c|}{ Sensitivity } \\
\hline & & Week 1 & Week 4 & Week 12 \\
\hline \multirow[t]{2}{*}{ RDT1 } & 200 parasites $/ \mu l$ & 100 & 100 & 100 \\
\hline & 2,000 parasites $/ \mu \mathrm{l}$ & 100 & 100 & 100 \\
\hline \multirow[t]{2}{*}{ RDT2 Pf (Pan) } & 200 parasites $/ \mu l$ & $100(22) \dagger$ & $100(75) \dagger$ & $100(12.5) \dagger$ \\
\hline & 2,000 parasites $/ \mu \mathrm{l}$ & $100(100)$ & $100(100)$ & 100 (80)\# \\
\hline \multirow[t]{2}{*}{ RDT3 } & 200 parasites $/ \mu \mathrm{l}$ & 100 & 100 & 100 \\
\hline & 2,000 parasites $/ \mu \mathrm{l}$ & 100 & 100 & 100 \\
\hline \multirow[t]{2}{*}{ RDT4 } & 200 parasites $/ \mu \mathrm{l}$ & 100 & 100 & 100 \\
\hline & 2,000 parasites $/ \mu \mathrm{l}$ & 100 & 100 & 100 \\
\hline \multirow[t]{2}{*}{ RDT5 } & 200 parasites $/ \mu \mathrm{l}$ & $55.5 *$ & $12.5 t$ & 37.5 \\
\hline & 2,000 parasites $/ \mu \mathrm{l}$ & 100 & 100 & 100 \\
\hline \multirow[t]{2}{*}{ RDT6 Pf (Pan) } & 200 parasites $/ \mu \mathrm{l}$ & $89(67)$ & $100(33)$ & $75(0)^{*}$ \\
\hline & 2,000 parasites $/ \mu \mathrm{l}$ & $100(89)$ & $100(86)$ & $100(80)$ \\
\hline \multirow[t]{2}{*}{ RDT7 } & 200 parasites $/ \mu l$ & 100 & 100 & $87.5 \#$ \\
\hline & 2,000 parasites $/ \mu \mathrm{l}$ & 100 & 100 & 100 \\
\hline \multirow[t]{2}{*}{ RDT8 } & 200 parasites $/ \mu \mathrm{l}$ & 100 & 100 & 100 \\
\hline & 2,000 parasites $/ \mu \mathrm{l}$ & 100 & 100 & 100 \\
\hline \multirow[t]{2}{*}{ RDT9 } & 200 parasites $/ \mu \mathrm{l}$ & 100 & 100 & $87.5 \#$ \\
\hline & 2,000 parasites $/ \mu \mathrm{l}$ & 100 & 100 & 100 \\
\hline \multirow[t]{2}{*}{ RDT10 Pf (Pan) } & 200 parasites $/ \mu \mathrm{l}$ & $100(0)^{*}$ & $100(0)^{*}$ & $100(33)$ \\
\hline & 2,000 parasites $/ \mu l$ & $100(100)$ & $100(100)$ & $100(100)$ \\
\hline
\end{tabular}

† Pan band was weakly reactive for two of three parasites at baseline. $\$$ Weakly reactive for all three parasites at baseline.

* Pan test negative for two of three parasites and weakly reactive for the one positive parasite at baseline.

\# Reactivity lost for one sample stored at $35^{\circ} \mathrm{C}$.

was tested for up to 46 weeks and RDT 10 (HRP2/ $\mathrm{pLDH})$ up to 26 weeks for samples at 1,000 parasites/ $\mu \mathrm{l}$ and up to 21 weeks for samples at 2,000 parasites/ $\mu$ l. However, at week 52, additional RDT products (RDTs 1,3 and 7) were positive for both parasite densities (Table 3b).

\section{Dried blood can identify faulty RDTs}

The ability of dried blood to identify faulty RDTs was demonstrated in the time series punctured RDT pouch tests. In these experiments, intact RDT pouches for both RDT2 and RDT5 retained their reactivity irrespective of whether they were stored at room temperature or $37^{\circ} \mathrm{C}$ up to the 4 weeks of testing. However, punctured tests stored at $37^{\circ} \mathrm{C}$ in a humidified incubator retained reactivity up to $30 \mathrm{hrs}$ and by week 2 were failing to show test bands with control bands very faint (additional file 2: Supplementary information).

\section{Discussion}

Malaria RDT performance can be adversely affected by storage at temperatures above the manufacturer's recommended range. According to manufacturer-provided product specifications, most RDTs require maximum storage temperatures of approximately $30^{\circ} \mathrm{C}$ above which test performance may be compromised, especially if tests are stored for prolonged periods at such temperatures. In sub-Saharan Africa, where RDTs are increasingly being used for malaria diagnosis, ambient temperatures are frequently above $30^{\circ} \mathrm{C}$ and, therefore, in peripheral health facilities where refrigeration is likely to be unavailable for cool storage of RDTs, high temperature exposure is expected to be common. The length of time a test has to be stored at the higher than the recommended storage temperatures before performance is affected is variable. Data from the WHO/FIND/CDC product evaluation indicate [12] most RDTs retain performance after prolonged periods above required storage temperatures while some others deteriorate and a minority improve in performance. It is not clear what level of heat exposure and for how long a test needs to be exposed for a definitive adverse effect on performance. Therefore, monitoring RDTs stored under such conditions is critical to an effective QC system. Such test monitoring could be achieved by positive controls that are regularly used to test for RDT performance. Unfortunately, because malaria RDTs require fresh or frozen parasitized blood and recombinantly produced positive control antigens are as yet unavailable, field monitoring has largely been ignored as a component of a comprehensive RDT quality assurance system. This is despite the importance of guaranteeing the quality of RDTs especially in peripheral health facilities where they are more likely to be used. In this study, parasite-infected blood dried in tubes was used as a simple method for preserving samples for QC of RDTs. The dried tube method has been successfully used for preserving samples for HIV antibody detection and was slightly modified to detect $P$. falciparum antigens in whole blood.

The use of dried P. falciparum-infected blood as QC samples appeared to be a feasible method for monitoring RDT performance. However, these results revealed that, for country-specific programmes standardization of the sample on the specific RDT to be monitored is critical to obtaining desired results. This test-specific standardization will not be desirable for product evaluation or when several products are being compared, since one standard will be required for all RDT products. While dried blood retained reactivity on four RDT brands irrespective of parasite isolate, parasite density, storage temperature and length of time stored, reactivity was lost on other RDTs dependent on these variables. Of note, detection of rehydrated dried blood by HRP2-specific tests or HRP2-specific test lines on combination tests was more consistent compared to pLDH bands. This result is similar to observations reported in the WHO/FIND/CDC RDT product evaluation that shows HRP2 tests to be more sensitive than 
Table 3 Extended stability results for dried Plasmodium falciparum 3D7 infected blood specimens

\begin{tabular}{|c|c|c|c|c|c|c|c|c|c|}
\hline \multirow[t]{2}{*}{ a) } & \multirow[t]{2}{*}{ 3D7 PD } & \multicolumn{2}{|l|}{ RDT 5} & \multicolumn{3}{|l|}{ RDT 2} & \multicolumn{3}{|c|}{ RDT 10} \\
\hline & & $\bar{C}$ & $T$ & $\bar{C}$ & Pan & $\mathrm{Pf}$ & $\bar{C}$ & Pan & $\mathrm{Pf}$ \\
\hline Baseline & $1000 \mathrm{p} / \mu \mathrm{l}$ & ++++ & ++++ & ++++ & ++++ & ++++ & ++++ & ++ & ++++ \\
\hline Week 12 & & ++++ & ++ & ++++ & ++ & ++++ & ++++ & + & +++ \\
\hline Week 21 & & ++++ & ++ & ++++ & ++ & ++++ & ++++ & + & +++ \\
\hline Week 26 & & ++++ & ++ & ++++ & ++ & ++++ & ++++ & + & +++ \\
\hline Week 35 & & ++++ & ++ & ++++ & ++ & ++++ & ND & & \\
\hline Week 38 & & ++++ & ++ & ++++ & ++ & +++ & ND & & \\
\hline Week 41 & & ++++ & ++ & ++++ & ++ & +++ & ND & & \\
\hline Week 46 & & ++++ & ++ & ++++ & ++ & +++ & ND & & \\
\hline \multirow[t]{2}{*}{ Week 52} & & ND & & ++++ & ++ & +++ & ND & & \\
\hline & & C & $\mathrm{T}$ & C & Pan & $\mathrm{Pf}$ & $C$ & Pan & $\mathrm{Pf}$ \\
\hline Baseline & $2000 \mathrm{p} / \mu \mathrm{l}$ & ++++ & ++++ & ++++ & ++++ & ++++ & ++++ & ++++ & ++++ \\
\hline Week 12 & & ++++ & ++++ & ++++ & +++ & ++++ & ++++ & ++ & ++++ \\
\hline Week 21 & & ++++ & ++++ & ++++ & ++ & ++++ & ++++ & ++ & +++ \\
\hline Week 26 & & ++++ & ++++ & ++++ & ++ & +++ & ND & & \\
\hline Week 35 & & ++++ & ++++ & ++++ & ++ & +++ & ND & & \\
\hline Week 38 & & ++++ & ++ & ++++ & ++ & +++ & ND & & \\
\hline Week 41 & & ++++ & ++ & ++++ & ++ & +++ & ND & & \\
\hline Week 46 & & ++++ & ++ & ++++ & ++ & +++ & ND & & \\
\hline Week 52 & & ND & & ++++ & ++ & +++ & ND & & \\
\hline \multirow[t]{2}{*}{ b) } & 3D7 PD & RDT 1 & & RDT 3 & & & RDT 7 & & \\
\hline & & $\bar{C}$ & $\mathrm{Pf}$ & $\mathrm{C}$ & PV & $\mathrm{Pf}$ & $\bar{C}$ & $T$ & \\
\hline \multirow[t]{2}{*}{ Week 52} & $1000 \mathrm{p} / \mu \mathrm{l}$ & ++++ & ++++ & ++++ & - & +++ & ++++ & +++ & \\
\hline & $2000 p / \mu l$ & ++++ & ++++ & ++++ & - & ++++ & ++++ & ++++ & \\
\hline
\end{tabular}

pLDH. Essentially, the reactivity of the rehydrated dried blood was test-dependent. For instance, at 200 parasites/ $\mu \mathrm{l}$, all three parasite isolates showed weak reactivity at baseline on RDT 5, a pLDH only test, while pLDH reactivity was lost for two of the three parasites on RDTs 6 and 10, both combination tests detecting HRP2 and pLDH. In addition to test-dependent differences, the parasite isolate also appeared to influence reactivity. At 200 parasites/ $\mu \mathrm{l}, \mathrm{PH} 1$ was likely being recognized close to its detection limit, which is conferred by the amount of HRP2 it produces. Irrespective of storage temperature and length of storage, $\mathrm{PH} 1$ at this parasite concentration was associated with more weak or lost reactivity than the other parasite isolates irrespective of whether HRP2 or pLDH was the target antigen.

Based on the parasite and test-dependent reactivity patterns, the use of dried blood requires careful characterization and standardization of the parasite isolate for the type of tests to be monitored. This should include the use of standard protocols for generation and dilution of validated and quality controlled samples. Strong baseline reactivity at a required parasite density may be critical to retaining dried blood reactivity after rehydration since weak baseline reactivity may suggest reactivity at the limit of detection of the test and slight intra-lot variations may result in loss of reactivity on some tests. Although this study did not identify any clear temperature-related loss of reactivity of dried blood, the loss of reactivity of $\mathrm{PH} 1$ at 200 parasites/ $\mu$ l stored only at $35^{\circ} \mathrm{C}$ for 12 weeks on RDTs 7 and 9 suggests a possible temperature effect and therefore prolonged storage should be at cool temperatures. This has implications for the practicality of dried blood stored at peripheral health facilities. One solution could be that dried blood is stored for shorter times at peripheral health facilities if refrigeration is unavailable or that supervisors take along during supervisory visits tubes containing dried samples stored at cool temperatures at a higher level health facility. This is supported by the retention of dried 3D7 reactivity for over 12 months. It was also demonstrated 
that failing tests could be identified using the dried blood. In addition to their utility as QC samples, dried blood panels could be developed into proficiency testing panels for assessing health workers' ability to perform RDTs and interpret results. Such a use could double as assessing the competency of health workers and test performance at the same time.

\section{Conclusions}

In the absence of recombinant positive control antigens, well-characterized, dried P. falciparum-infected blood in tubes can be used as QC samples for monitoring the quality of RDTs. The samples used should be well characterized and standardized for their baseline reactivity on a range of performance acceptable RDTs. Field testing of this methodology is necessary to assess applicability under field conditions.

\section{Additional files}

Additional file 1: Supplementary information. Dried Tube Experiments. Colour-coded results for 10 RDT brands. Results are shown as relative band intensities for control and test bands.

Additional file 2: Supplementary information. Punctured RDT Testing. Time series experiments using punctured RDT pouches. Test results for intact and punctured RDTs 2 and 5 stored at room temperature or $37^{\circ} \mathrm{C}$ and tested over time.

\section{Competing interests}

The authors declare that they have no competing interests.

\section{Authors' contributions}

MA conceived and designed the study. MA and JP carried out the experiments. MA, JP and JWB analysed the data and wrote the manuscript. All authors read and approved the final manuscript.

\section{Acknowledgements}

We thank Bharat Parekh, Hetal Patel and Mireille Kalou of the International Laboratory Branch, Division of Global HIV/AIDS, Centers for Disease Control and Prevention, for their invaluable assistance and useful discussions.

Received: 10 May 2012 Accepted: 2 July 2012

Published: 23 July 2012

\section{References}

1. WHO: Guidelines for the Treatment of Malaria. Second Editionth edition. Geneva: World Health Organization; 2010.

2. WHO: World Malaria Report 2010. Geneva: World Health Organization; 2010

3. Ceesay SJ, Casals-Pascual C, Erskine J, Anya SE, Duah NO, Fulford AJ, Sesay SS, Abubakar I, Dunyo S, Sey O, Palmer A, Fofana M, Corrah T, Bojang KA, Whittle HC, Greenwood BM, Conway DJ: Changes in malaria indices between 1999 and 2007 in The Gambia: a retrospective analysis. Lancet 2008, 372:1545-1554

4. Mmbando BP, Vestergaard LS, Kitua AY, Lemnge MM, Theander TG, Lusingu JP: A progressive declining in the burden of malaria in north-eastern Tanzania. Malar J 2010, 9:216.

5. Steketee RW, Sipilanyambe N, Chimumbwa J, Banda JJ, Mohamed A Miller J, Basu S, Miti SK, Campbell CC: National malaria control and scaling up for impact: the Zambia experience through 2006. Am J Trop Med Hyg 2008, 79:45-52.

6. McMorrow ML, Masanja MI, Abdulla SM, Kahigwa E, Kachur SP: Challenges in routine implementation and quality control of rapid diagnostic tests for malaria-Rufiji District, Tanzania. Am J Trop Med Hyg 2008, 79:385-390.
7. Nankabirwa J, Zurovac D, Njogu JN, Rwakimari JB, Counihan H, Snow RW, Tibenderana JK: Malaria misdiagnosis in Uganda-implications for policy change. Malar J 2009, 8:66

8. Bell D, Wongsrichanalai C, Barnwell JW: Ensuring quality and access for malaria diagnosis: how can it be achieved? Nat Rev Microbiol 2006, 4(Suppl):S7-S20

9. D'Acremont V, Malila A, Swai N, Tillya R, Kahama-Maro J, Lengeler C, Genton B: Withholding antimalarials in febrile children who have a negative result for a rapid diagnostic test. Clin Infect Dis 2010, 51:506-511.

10. Harvey SA, Jennings L, Chinyama M, Masaninga F, Mulholland K, Bell DR: Improving community health worker use of malaria rapid diagnostic tests in Zambia: package instructions, job aid and job aid-plus-training. Malar J 2008, 7:160.

11. McMorrow ML, Masanja MI, Kahigwa E, Abdulla SM, Kachur SP: Quality assurance of rapid diagnostic tests for malaria in routine patient care in rural Tanzania. Am J Trop Med Hyg 2010, 82:151-155.

12. Thiam S, Thior M, Faye B, Ndiop M, Diouf ML, Diouf MB, Diallo I, Fall FB, Ndiaye $\mathrm{J}$, Albertini A, Lee E, Jorgensen P, Gaye O, Bell D: Major reduction in anti-malarial drug consumption in Senegal after nation-wide introduction of malaria rapid diagnostic tests. PLoS One 2011, 6:e18419.

13. Malaria rapid diagnostic test performance - Results of WHO product testing of malaria RDTs: Round 2 (2009). Geneva: World Health Organization; 2010.

14. Chiodini PL, Bowers K, Jorgensen P, Barnwell JW, Grady KK, Luchavez J, Moody AH, Bell D: The heat stability of Plasmodium lactate dehydrogenase-based and histidine-rich protein 2-based malaria rapid diagnostic tests. Trans R Soc Trop Med Hyg 2007, 101:331-337.

15. Kyabayinze DJ, Tibenderana JK, Odong GW, Rwakimari JB, Counihan H: Operational accuracy and comparative persistent antigenicity of HRP2 rapid diagnostic tests for Plasmodium falciparum malaria in a hyperendemic region of Uganda. Malar J 2008, 7:221.

16. Mtove G, Nadjm B, Amos B, Hendriksen IC, Muro F, Reyburn H: Use of an HRP2-based rapid diagnostic test to guide treatment of children admitted to hospital in a malaria-endemic area of north-east Tanzania. Trop Med Int Health 2011, 16:545-550.

17. D'Acremont V, Kahama-Maro J, Swai N, Mtasiwa D, Genton B, Lengeler C Reduction of anti-malarial consumption after rapid diagnostic tests implementation in Dar es Salaam: a before-after and cluster randomized controlled study. Malar J 2011, 10:107.

18. Ravaoarisoa E, Zamanka H, Fusai T, Bellalou J, Bedouelle H, MercereauPuijalon O, Fandeur T: Recombinant antibodies specific for thePlasmodium falciparumhistidine-rich protein 2. MAbs 2010, 2:416-427.

19. Lon CT, Alcantara S, Luchavez J, Tsuyuoka R, Bell D: Positive control wells: a potential answer to remote-area quality assurance of malaria rapid diagnostic tests.Trans R Soc Trop Med Hyg 2005, 99:493-498.

20. Versteeg I, Mens PF: Development of a stable positive control to be used for quality assurance of rapid diagnostic tests for malaria. Diagn Microbiol Infect Dis 2009, 64:256-260.

21. Parekh BS, Anyanwu J, Patel H, Downer M, Kalou M, Gichimu C, Keipkerich BS, Clement N, Omondi M, Mayer O, Ou CY, Nkengasong JN: Dried tube specimens: a simple and cost-effective method for preparation of HIV proficiency testing panels and quality control materials for use in resource-limited settings. J Virol Methods 2010, 163:295-300

22. WHO: Methods Manual for Product testing of Malaria Rapid Diagnostic Tests. Version 3. WHO/TDR, FIND, CDC; 2010.

doi:10.1186/1475-2875-11-239

Cite this article as: Aidoo et al.: Dried Plasmodium falciparum-infected samples as positive controls for malaria rapid diagnostic tests. Malaria Journal 2012 11:239. 